\title{
A Biofluidic Random Laser Cytometer for Biophysical Phenotyping of Cell Suspensions
}

Jijun $\mathrm{He}^{\mathrm{a}^{\dagger}}$, Shuhuan $\mathrm{Hu}^{\text {bcg }}{ }^{\dagger *}$, Jifeng Ren ${ }^{\mathrm{b}}$, Xin Cheng ${ }^{\mathrm{a}}$, Zhijia Hu${ }^{\mathrm{de}}$, Ning Wang ${ }^{\mathrm{f}}$, Huangui Zhang ${ }^{\mathrm{cg}}$, Raymond H. W. Lam ${ }^{\mathrm{b}}$, Hwa-Yaw Tam ${ }^{\mathrm{a}}$

a Department of Electrical Engineering, The Hong Kong Polytechnic University, Kowloon, Hong Kong, China

${ }^{\mathrm{b}}$ Department of Mechanical and Biomedical Engineering, City University of Hong Kong, Kowloon, Hong Kong, China

c BGI-Shenzhen, Shenzhen 518083, Guangdong, China

${ }^{\mathrm{d}}$ School of Instrument Science and Opto-Electronics Engineering, Hefei University of Technology, Hefei 230009, China

e Aston Institute of Photonic Technologies, Aston University, Birmingham B4 7ET, UK

${ }^{\mathrm{f}}$ National Engineering Laboratory for Fiber Optic Sensing Technology, Wuhan University of Technology, Wuhan, China

${ }^{g}$ Guangdong High-throughput Sequencing Research Center, Guangdong, China $\dagger$ these authors contributed equally to this work * correspondence should be addressed to shuhuanhu2-c@my.cityu.edu.hk 


\begin{abstract}
Phenotypic profiling of single floating cells in liquid biopsies is the key to the era of precision medicine. Random laser in biofluids is a promising tool for the label-free characterization of the biophysical properties due to the high brightness and sharp peaks of the lasing spectra, yet previous reports were limited to the random laser in solid tissues with dense scatterings. In this report, a random laser cytometer is demonstrated in an optofluidic device filled with gain medium and human breast normal/cancerous cells. The multiple light scattering induced by the micro-scale human cells promotes the random lasing and influences the lasing properties in term of laser modes, spectral wavelengths, and lasing thresholds. A sensing strategy based on analyzing the lasing properties to determine both the whole-cell and the subcellular biophysical properties of the cells and the malignant alterations of the cell suspensions are successfully detected. Our results provide a new approach to designing a label-free biophysical cytometer based on optofluidic random laser devices, which is advantageous for further research in the field of random laser bio-application.
\end{abstract}

\title{
Key Words
}

Optofluidics, Random Laser, Cytometer, Phenotype, Cell Suspension, Biophysics, Cancer Cells. 
Optofluidics that synergistically combine optics and microfluidics are the keys to the next generation cytometers. ${ }^{1,2}$ Due to the flexibility of microfluidic system, optofluidic devices show great promise for numerous applications, particularly for bio-sensing platform. ${ }^{3-6}$ One of the most remarkable optofluidic devices is biofluidic laser, which integrates biological liquid suspensions and optical cavities into compact optofluidic system. ${ }^{7}$ The lasing properties such as intensity, spectrum, and threshold are sensitive to specific biomarkers such as biophysical alternations of the samples, which provided an label-free approach to the liquid biopsy diagnostics. ${ }^{7}$ Compared with the traditional optofluidic bio-sensing devices using fluorescence (i.e. spontaneous emission) as the sensing signals, the biofluidic laser shows several merits. First, the laser signal exhibits much higher peak intensity with a very narrow linewidth (normally, below one nanometer), and delivers higher signal-to-noise ratio (SNR) hence the signal is easier to be detected and determined. ${ }^{7}$ Second, the laser feedback is an innate amplifier for optical signal variation induced by the biophysical alternations of the liquid biopsies, endowing the biofluidic laser with a high sensitivity. ${ }^{8}$ Third, the unique threshold of lasing in a biofluidic system is highly correlated with the biophysical state, which is a sensitive indicator for the biomarker detection. Therefore, biofluidic lasers have attracted great interest and a lot of attention has been devoted to this topic. ${ }^{7}$ To date, biofluidic random lasers have been demonstrated in various configurations wherein the laser feedbacks are provided by optical cavities of various configurations, such as highQ ring resonators, ${ }^{8-10}$ Fabry-Perot cavities, ${ }^{11,12}$ and distributed feedback gratings ${ }^{13,14}$. These well-defined optical cavities generally require precise design and fabrication. For example, Fabry-Perot cavity consists of a pair of mirrors which need to be perfectly aligned to each other. This would increase the manufacturing costs and difficulty, which limits the developing of the biofluidic lasers. ${ }^{11}$ Therefore, there is a need to achieve laser feedback in optofluidic devices via a facile approach.

A cavity-free optofluidic laser has been proposed by Shivakiran Bhaktha and colleagues in 2012. ${ }^{15}$ In their work, the conception of random laser was firstly introduced into the optofluidic devices to achieve laser feedback without optical cavities. Different from 
traditional lasers, laser feedback in random lasers is provided by multiple light scattering in disordered systems containing gain medium. ${ }^{16,17}$ This unique laser feedback renders many advantages to random lasers that include ease of fabrication and controllable lasing properties (e.g. intensity, wavelength, threshold, and direction). ${ }^{18}$ Although several studies have investigated the random lasing properties in optofluidic systems, ${ }^{19,20}$ applying random lasers in biofluidics for bio-sensing is still very challenging, mostly due to the limited understanding of the intercellular and intracellular biophysical properties of the biopsies ${ }^{8}$. However, by carefully choosing biophysically well-defined bio-samples, the biological tissues have already been demonstrated that they are suitable media to induce the generation of random lasing. In 2004, Polson and co-researchers firstly reported that random lasing can be observed in gain-medium-infiltrated human colon tissues that was composed of highly hierarchical structures. ${ }^{21}$ Thereafter, the random lasing action has been achieved based on other biological tissues, such as chicken breast, ${ }^{22}$ bone tissue ${ }^{23}$ and butterfly wings ${ }^{24,25}$, in which solid niche (e.g. in bone tissue) or fibers (e.g. in chicken breast and butterfly wings) can provide strong scatterings and form laser cavities. Clinically, it has been proposed that random lasers have the potential application for tumor detection because the lasing properties are highly sensitive with the malignant alternations of the tumor tissues. ${ }^{26}$ It was found that the random laser induced by the cancerous tissue exhibited more laser modes than that induced by the healthy tissue, which might be due to the more heterogeneous cancerous tissue that provides more laser cavities. ${ }^{21}$ For instance, Wang et al. has demonstrated that the human breast tumor tissues with different malignancy grades were highly correlated with the specific random laser spectra and thresholds. $^{26}$

However, previous demonstrations of random laser in biosensing on solid tissues suffered from the availability of solid biopsies in clinical practices. Comparing to solid tissues that need surgical cutting from the disordered organs, liquid biopsies are much easier to obtain in clinical practices. Importantly, liquid biopsies, such as circulating tumor cells (CTCs) that shed from the primary tumors, provide prognostic biomarkers at the very initiation of the disease. Thus, it is of great value to apply novel biosensing 
techniques to liquid biopsy prognostics. Technically, random laser detection at the subcutaneous vessel is inherently noninvasive, which could be developed in vitro by the biofluidic platform embedded with random laser peripheries. The advantages of applying random laser in liquid biopsy prognostics are considerable. As far as we are aware, no study on the application of random laser to biological liquids in vitro or in vivo has been conducted.

In the present work, we demonstrate a novel biofluidic random laser (BFRL) cytometer for the characterization of cell suspensions. Suspensions of human breast epithelial cells (MCF-10A) and human breast cancerous cells (MCF-7 and MDA-MB-231) are used. The BFRL is achieved by lasing in a geometrically well-defined microfluidic channel filled with cell suspensions added with gain medium. The lasing properties are distinct when different types of cells are introduced, which enables the identification of the different cell types via analyzing the random lasing spectra. Careful analysis reveals the roles of biophysical alternations of the cell suspensions in determining the specificity of the laser spectra. To the best of our knowledge, this is the first demonstration of using BFRL to distinguish normal and cancerous cells in liquid suspensions, which paves the way towards novel cytometers for liquid biopsy prognostics.

\section{Experiment Section}

\section{Preparation of cells}

Human breast epithelial cells (MCF-10A), breast cancerous cells (MCF-7 and MDAMB-231) were obtained from ATCC (Manassas, VA). MCF-10A cells were cultured in the Mammary Epithelial Growth Medium (MEGM; CC-3150, Lonza, Walkersville, MD) added with $0.4 \%(\mathrm{v} / \mathrm{v})$ bovine pituitary extract (BD, Franklin Lakes, NJ), $0.1 \%$ (v/v) human epithelial growth factor (hEGF; Cell Signaling Technology, Beverly, MA), $0.1 \%(\mathrm{v} / \mathrm{v})$ hydrocortisone (Sigma-Aldrich, St. Louis, MO), $0.1 \%$ (v/v) insulin (SigmaAldrich) and $0.1 \%(\mathrm{v} / \mathrm{v})$ of a reagent mixed with $30 \mathrm{mg} / \mathrm{ml}$ gentamicin and $15 \mu \mathrm{g} / \mathrm{ml}$ amphotericin (GA-1000, Lonza). MCF-7 cells were cultured in a high-glucose 
Dulbecco's modified Eagle's medium (DMEM; Invitrogen, Carlsbad, CA) with the supplement of $10 \%$ fetal bovine serum (Atlanta Biological, Atlanta, GA), $0.5 \mu \mathrm{g} / \mathrm{ml}$ fungizone (Invitrogen, Carlsbad, CA), $5 \mu \mathrm{g} / \mathrm{ml}$ gentamicin (Invitrogen), 100 units $/ \mathrm{ml}$ penicillin, and $100 \mu \mathrm{g} / \mathrm{ml}$ streptomycin. MDA-MB-231 cells were cultured in DMEMF12 (Invitrogen) added with $10 \%$ fetal bovine serum and 100 units $/ \mathrm{ml}$ penicillin. All cells were cultured at $37{ }^{\circ} \mathrm{C}$ with $\sim 100 \%$ humidity and $5 \% \mathrm{CO}_{2}$ in an incubator. To obtain suspended cells for the experiments, we used $0.25 \%$ trypsin-EDTA in phosphate buffered saline (PBS) to re-suspend the cells, following by centrifuge and replacement of fresh culture media. The cells were then diluted to the target cell density $\left(\sim 2 \times 10^{6}\right.$ cells $/ \mathrm{ml}$ ) by adding additional culture media and $2.5 \mathrm{mM}$ Rhodamine 640 dye before injected into the optofluidic device. Rhodamine 640 is commonly used in flow cytometry for labelling and it causes minimal influences to the suspended cells. ${ }^{27}$ All the experiments were conducted within $2 \mathrm{hr}$ after the cells were suspended.

\section{Fabrication of optofluidic device}

The optofluidic device is fabricated by standard photolithography based on the replica molding of elastomeric polydimethylsiloxane (PDMS) (Sylgard-184, Dow Corning, Midland, MI). First, a $35 \mu \mathrm{m}$ thick layer of negative photoresist (SU8-2025, MicroChem, Westborough, MA) with a zigzag channel was designed and patterned on a silicon wafer. The mold master was the silanized with vaporized (tridecafluoro1,1,2,2,-tetrahydrooctyl)-1-trichlorosilane (Sigma-Aldrich, St. Louis, MO) in a vacuum chamber. The PDMS substrate containing microchannels was fabricated by the soft lithography procedures. The PDMS substrate was then bonded onto a glass slide (Citoglas, Jiangsu, China) by oxygen plasma treatment.

\section{Random lasing measurement}

The experimental set-up for random lasing measurement follows our previous study. ${ }^{28}$ The pump source was a Q-switch Nd:YAG pulse laser (Continuum, Inlite II-50), operating at $532 \mathrm{~nm}$ wavelength with $6 \mathrm{~ns}$ pulse duration and $10 \mathrm{~Hz}$ repetition rate. A power meter (Coherent, LabMax-Top) equipped with a pyroelectric energy sensor was 
used to determine the pump laser power. The laser beam was focalized to the front surface of the BFRL into a line of 2.5-cm long and 1-mm wide, and parallel to the microchannel of the BFRL. The random lasing from the BFRL was collected by a multimode fiber with 50- $\mu \mathrm{m}$ core diameter (Thorlabs, GIF50C) located by the side of the opofluidic chip and directed to a spectrometer (Ocean Optics, HR4000) with measurement resolution of $0.1 \mathrm{~nm}$. The expose time for each spectrum is set to be $1 \mathrm{~s}$ to reduce the intensity fluctuation of the random lasing.

\section{Morphology characterization}

FEI Quanta 450 FEG scanning electron microscope (SEM, Dawson, NE) at a scanning voltage of $5 \mathrm{kV}$ and a spot size of 4.0 was applied to capture the images of the optofluidic channel at a tilt angle of $45^{\circ}$.

The cells were stained using immunofluorescence staining method at a floating state. To stain floating cells, the cells were first resuspended by using $0.25 \%$ trypsin-EDTA in phosphate buffered saline (PBS; Sigma-Aldrich, St. Louis, MO). The cells were fixed with $4 \%$ paraformaldehyde (PFA, Sigma-Aldrich, St. Louis, MO) in phosphate buffered saline (PBS, Sigma-Aldrich, St. Louis, MO) for $10 \mathrm{~min}$ and then treated with $0.3 \%$ Triton X-100 in PBS for 10 min. We applied $10 \%$ goat serum for $1 \mathrm{hr}$ to avoid non-specific binding of the staining molecules in the next steps. The Lamin-A primary antibody (Abcam, Cambridge, MA) was applied for $1 \mathrm{hr}$. The cytoskeletal actin was stained with Alexa-555 conjugated phalloidin (Life Technologies, Carlsbad, CA) followed by staining the nucleus with $0.1 \%$ Hoechst 33342 (Sigma-Aldrich, St. Louis, MO) for $10 \mathrm{~min}$. And the stained images were obtained by using laser confocal microscope (TCS SP8 Confocal Microscope, Leica, Germany). Bright field imaging is conducted by a phase-contrast inverted microscope (TE300, Nikon, Tokyo, Japan) and an sCMOS microscope camera (Zyla, Andor, Belfast, UK) was applied to capture highresolution images $(\sim 570 \mathrm{~nm} / \mathrm{pixel})$.

\section{Statistics}

$p$-values were calculated using the Student's $t$-test in Excel (Microsoft, Seattle, WA). 


\section{Results and Discussion}

\section{Experimental set-up}

The optofluidic device is composed of a zigzag channel, as shown in the SEM image in Figure 1a. The width of the channel is $400 \mu \mathrm{m}$ and the total length of the channel is $3 \mathrm{~cm}$. The design of the zigzag shape is to promote random lasing, as it has been reported that the channels with multi-scattering structures for light can enhance the efficiency of random lasing and reduce the lasing threshold. ${ }^{15,20,29}$ By injecting the mixture of the cells with concentration of $2 \times 10^{6}$ cells $/ \mathrm{ml}$ and $2.5 \mathrm{mM}$ Rhodamine 640 dye into the zigzag channel, the BFRL was filled with liquid suspensions of MCF-10A, MCF-7 or MDA-MB-231 cells. In order to fully excite the BFRLs, a side-pumping configuration is employed, as shown in Figure 1b. The pump source used in our experiments is a Nd:YAG pulse laser $(532 \mathrm{~nm}, 10 \mathrm{~Hz})$. The pump laser beam was focalized by a cylindrical lens to a line of $2.5-\mathrm{cm}$ long and $1-\mathrm{mm}$ wide. The pump laser beam was adjusted precisely to cover the channel of BFRL to achieve uniform pumping. The zigzag channel performed as a waveguide hence the random lasing generated in the BFRL would be guided to emit through the exit of the channel. A multimode fiber was placed closed to the exit of the optofluidic channel to collect and transmit the random lasing spectra to the spectrometer. The random lasing properties of the BFRLs were then measured and analyzed under the same experiment configuration.

\section{Evolution of the emission spectra and random lasing}

Figure 2a shows the evolution of emission spectra recorded from the BFRL filled with MCF-10A cell suspensions excited at different pump powers. The intensity of the collected emission spectra increases as the pump power increases. More importantly, only spontaneous emissions with broad spectra between $600 \mathrm{~nm}$ and $660 \mathrm{~nm}$ were observed when the pump power was low, while a narrow emission band at $\sim 610 \mathrm{~nm}$ manifested itself in the emission spectra when the pump power exceeded a certain 
threshold. These discrete sharp peaks emerging on the top of the narrow emission band were features of coherent random lasing and the corresponding pump power threshold was the lasing threshold. ${ }^{30,31}$ The full-width-half-maximum (FWHM) of the main sharp peak was measured to be $0.35 \mathrm{~nm}$, as shown in the insert of Figure 2a. Similar random lasing characteristics have also been observed in the emission spectra recorded from the BFRL filled with MCF-10A and MDA-MB-231 cells (see Figure $2 b$ and c). The corresponding FWHM of the sharp peaks are $0.41 \mathrm{~nm}$ and $0.27 \mathrm{~nm}$, respectively. It is worth noting that, for the cell suspensions with different types of cells, the intensity and profile of the random laser spectra were different. Especially, the lasing spectra obtained from the BFRL filled with MCF-10A shows an almost smooth profile with weak lasing peaks. Such feature indicates the incoherent random lasing behavior due to the low scattering strength. ${ }^{17}$

\section{Lasing mechanism of the BFRL filled with the cells}

To get a better understanding of the origins of these spectral differences, we summarized the lasing mechanism of the BFRL and clarified these differences accordingly, see the schematic in Figure 3. Excited by the incident laser beam, fluorescent dyes in the zigzag channel worked as gain medium and emitted spontaneous emission. The existing of the cells provided large amount of scattering events for the emission light since the refractive index of the cells are different with the solution $(\sim 1.40$ versus $\sim 1.35) .{ }^{32}$ The light path was increased by the zigzag shape of the channel, which dramatically increased the scattering events furthermore. All these scattering provided enough amplifications for the emission light to generate the random lasing. The multi-scattering events were the keys for the formation of random lasing and the different lasing properties reflected the different scattering conditions. In fact, highly disordered biological system that intensely scattered light displayed the typical random lasing spectra, which is characterized by multiple sharp peaks on the base spectra, as shown in Figure $2 .{ }^{26}$ Moreover, the emission spectra of the BFRL with or without cells were quite different: for the optofluidic device without cells, only amplified 
spontaneous emission (ASE) can be observed in the emission spectrum. This means that the random lasing arises from the multiple light scattering provided by the cells. To describe the scattering strength provided by the cells, the scattering mean free path $l_{\mathrm{s}}$ is defined as the average distance that light travels between two consecutive scattering events. This parameter is determined as ${ }^{33,34}$

$$
l_{\mathrm{s}}=\frac{1}{\rho \sigma_{\mathrm{s}}}
$$

where $\rho$ is the particle density of the cell suspension and $\sigma_{S}$ is the scattering cross section of an individual cell. The scattering cross section $\sigma_{s}$ is a parameter associated with the size and refractive index of the cells, given by ${ }^{35,36}$

$$
\sigma_{s}=\frac{8}{3} x^{4}\left(\frac{n^{2}-1}{n^{2}+2}\right)^{2} \sigma_{g},
$$

where $x=\pi d / \lambda$ is the so-called size parameter, $n$ is the refractive index of the cells, $d$ is the diameter of an individual cell, $\lambda$ is the light wavelength, and $\sigma_{g}=\pi d^{2} / 4$ is the geometrical cross section. Thus, equation 1 can be rewritten as

$$
l_{\mathrm{s}}=\frac{3 \lambda^{4}}{2 \pi^{5} d^{6} \rho}\left(1+\frac{3}{n^{2}-1}\right)^{2},
$$

The light scattering strength in BRFL is mainly determined by the biophysical properties (size and refractive index) of the cells and the cell densities. Particularly, by analyzing the sensitivities of the three parameters, we found that the light scattering is mostly related to the single cell scattering properties. (See Supporting Information, sensitivity analysis)

\section{Biophysical properties and the lasing threshold}

As the biophysical properties of the cells strongly influence the light scattering properties, different lasing properties could perform as the indicator of the different biophysical properties of the suspended cells. Since the density of the particle density $\rho$ of the three cell suspensions was the same $\left(2 \times 10^{6}\right.$ cells $\left./ \mathrm{ml}\right)$. The main parameter which influences the scattering mean free path $l_{\mathrm{s}}$ is the diameter of the cells $d$ and refractive index of the cells $n$. The morphologies of the normal cells (MCF-10A) and 
the cancerous cells (MCF-7 and MDA-MB-231) are shown in the optical images in Figure 4a. The diameter of the MCF-10A cells $(14.23 \pm$ SE $0.35 \mu \mathrm{m})$ were much smaller while the MCF-7 cells and MDA-MB-231 cells were almost of the same size (20.36 \pm SE $0.28 \mu \mathrm{m}$ for MCF-7 and 19.75 \pm SE $0.56 \mu \mathrm{m}$ for MDA-MB-231), as shown in Figure 4b. Moreover, it has been reported that the refractive index of the normal cells is lower than that of the cancerous cells. ${ }^{37}$ According to Equation 3, the scattering strength provided by MCF-10A cells is much weaker than that provided by the two types of cancerous cells. The BFRL filled with MCF-10A cells shows less sharp peaks on the lasing spectra due to the low scattering strength provided by MCF-10A cells. Additionally, the intensity of the BFRL filled with MCF-10A cells is much weaker than that with other cell types (Figure 4c). These spectral differences provide important features to distinguish cells based on their biophysical properties, and potentially is a powerful approach for label-free cell identification.

To quantitatively analysis the influence of biophysical properties of the cells on the lasing properties, we extract the intensities of the main lasing peak of the BFRL as a function of the pump power (Figure 4d). Nonlinear dependences of the lasing intensities on the pump power were observed in the BFRL with all the three types of cells, exhibiting lasing threshold behavior. The lasing thresholds of the BFRL are determined to be $80.4 \pm \mathrm{SE} 1.8 \mathrm{~nJ} / \mathrm{mm}^{2}$ (for MCF-7), $83.8 \pm \mathrm{SE} 2.5 \mathrm{~nJ} / \mathrm{mm}^{2}$ (for MDA-MB-231), and $96.2 \pm \mathrm{SE} 1.6 \mathrm{~nJ} / \mathrm{mm}^{2}$ (for MCF-10A), respectively. The BFRL filled with MCF$10 \mathrm{~A}$ cells exhibits the largest lasing threshold and the smallest slope value of the lasing intensity versus the pump energy. This is because the scattering strength provided by the normal cells (MCF-10A) is much weaker than that provided by the cancerous cells (MCF-7 and MDA-MB-231). As a result, the pathlength for the emission light prorogation in the BFRL filled with MCF-10A is relatively short, which means the emission light need experience more amplification to lase. Thus, the MCF-10A cells with small cell size and low refractive index can be easily distinguished based on the lasing threshold of the BFRL.

Besides the size and the refractive index of the cells, the cell density also shows an important influence on the laser thresholds because the scattering strength of the BFRL 
are highly dependent on the filled cell density. To verify this influence, a set of experiments with cell density varied from $7 \times 10^{3}$ cells $/ \mathrm{ml}$ to $8 \times 10^{7}$ cells $/ \mathrm{ml}$ was carried out, results are shown in Figure S1. Indeed, the laser threshold varies as the cell density changes. However, under the same cell density, the laser threshold of the BFRL filled with MCF-10A/MCF-7 is always the highest/lowest among the three BFRLs, which is consistent with the above-mentioned discussion. In the rest of this study, the cell density is set to be $2 \times 10^{6}$ cells $/ \mathrm{ml}$. Under this cell density, the laser threshold values are low and the differences between the laser threshold values of the BFRL with different cell types are distinct. In our study, the cell density is set to be $2 \times 106$ cells $/ \mathrm{ml}$. Under this cell density, the laser threshold values are low and the differences between the laser threshold values of the BFRL with different cell types are distinct. Detailed analysis and discussion can be found in the Supporting Information.

\section{The lasing wavelength shift of the BFRL and subcellular biophysical properties}

The lasing threshold is an effective indicator related to the whole cell biophysical property - cell size and refractive index, and we used these indicators to successfully draw a distinction between the normal cells and the cancerous cells. However, the lasing threshold of the BFRL filled with the cancerous cells are similar to each other. To distinguish the MCF-7 cells from the MDA-MB-231 cells, we carefully compared the lasing spectra, as shown in Figure 5a, of the BFRL filled with the cancerous cells at the same pump power of $126 \mathrm{~nJ} / \mathrm{mm}^{2}$. The intensities of these two lasing spectra are similar. It is observed that the lasing wavelength of the BFRL filled with MCF-7 cells has a 2.3 \pm SE $0.2 \mathrm{~nm}$ blue-shift with respect to that of the BFRL filled with MDA-MB-231 cells. This lasing wavelength shift phenomenon has been observed in previous studies, wherein the scattering strength of the random laser system is variable. ${ }^{29,36,38-40}$ The corresponding mechanism can be illustrated based on analyzing another important biophysical property such as refractive index. For example, Hu et al shows that 0.011

refractive index variation could result in a spectral shift up to $18.5 \mathrm{~nm} .{ }^{36} \mathrm{In}$ our case, the refractive index of the MCF-7 cells (1.401) is higher than that of MDA-MB-231 cells (1.399), ${ }^{32}$ and it is very likely that one of the main sources for the $2.3 \mathrm{~nm}$ spectral 
shift is coming from the small difference (0.002) of refractive index (also see the sensitivity analysis section in Supporting Information). Thus random laser delivered a very high precision quantification of refractive index variations of biological samples, which could be used to develop high precision cytometers. Theoretically, high refractive index and short light wavelength will result in a short scattering mean free path, leading to more scattering events, see Equation 3. The BFRL system with strong scattering strength would trap light effectively and increase the light propagation path inside the system. The longer the light path is, the stronger amplification the emission light experiences. As a result, the emission light at short wavelength band would be preferentially amplified to lase as the scattering strength of the BFRL increases. Consequently, the MCF-7 and MDA-MB-231 cells can be distinguished based on this spectral blue-shift observation.

According to the discussion in the previous section, the BFRL filled with MCF-10A cells shows the weakest scattering strength due to the small cell size. Therefore, its lasing wavelength should have a red-shift compared with the other two samples. However, it can be seen from Figure $5 b$ that the lasing wavelength of the BFRL filled with MCF-10A cells is almost the same with that of the BFRL filled with MDA-MB231 cells at high pump power. This means there are other effects that play an important role in the spectral shift. To interpret this observation, we take into account the subcellular biophysical properties of the cells. Figure 5c-e shows the core-shell structure of three different cell lines. Clear shell subcellular structure can be observed in the normal cells (MCF-10A). The cell cortex (represented by the cortical actin, stained in red) and the nuclear lamina (represented by Lamin-A, stained in green) are the two structural shells of the cell. Nevertheless, due to the epithelial-mesenchymal transition (EMT) during cancer progression, the rigid structural proteins are down regulated and thus the shell structure of the invasive cancerous cells (MDA-MB-231) become less obvious. ${ }^{41}$ In order to show how the subcellular biophysical properties of the cells affect the lasing properties, we present the schematic of a typical light path loop in BFRL, as shown in Figure 5f. The light path in the BFRL consists of two parts, one is the light traveling in the gain medium, another is the light traveling inside the 
cells. The latter one would be manipulated by the subcellular biophysical properties of the cells, which further influence the lasing properties of the BFRL. ${ }^{42-44}$ Since the gain medium is outside of the cells, the light traveling inside the cells cannot be amplified. For normal cells (MCF-10A), the hierarchical subcellular structure would scatter the light out and prevent the light traveling inside the cells. This light scattering strength is inversely proportional to the light wavelength. It means that the emission light at short wavelength band travels less pathlength inside the cells and avoids the loss, resulting in a blue-shift of the lasing spectra. For the MDA-MB-231 cells, the scattering strength provide by the cortical shell and the lamina shell is relatively week because of the homogenized shell structure. ${ }^{45,46}$ Thus the light inside the cancer cells cannot be amplified and the lasing wavelength of the BFRL filled with the MDA-MB-231 cells shows no obvious different from the case with the MCF-10A cells, which deviates from the scattering theory. Moreover, this subcellular biophysical structure difference could also be one of the main contributions to the spectral shift between the BFRL filled with MCF-10A and MDA-MB-231 cells.

\section{Power Fourier transform analysis and laser cavity size in the BFRL}

Equivalent lasing cavity in the BFRL is a comprehensive indicator of the light scattering properties of the cell suspensions, which could be extracted from the lasing spectra. The equivalent lasing cavity is thus highly related to the biophysical properties of the suspended cells, such as cell size, refractive index, subcellular structure, etc.. We performed the power Fourier transform (PFT) analysis to verify the sizes of the lasing cavities excited in the BFRL. ${ }^{28}$ Figure 6 shows the PFT results (in $k=2 \pi / \lambda$ space) of the random lasing spectra recorded in the BFRL at the same pump power of 128 $\mathrm{nJ} / \mathrm{mm}^{2}$. For each sample, five spectra from five identical measurements are analyzed to verify the repeatability. The peaks in the PFT results corresponds to the Fourier components $p_{m}=m n L_{c} / \pi$, where $m$ is the order of the Fourier harmonic, $n$ is the refractive index of the liquid medium of the BFRL, and $L_{\mathrm{c}}$ is the pathlength of the lasing cavity. The fundamental Fourier components $p_{m=1}$ (i.e. the optical pathlength) of the three PFT results are determined to be $29.0 \pm$ SE $0.5 \mu \mathrm{m}, 12.1 \pm$ SE $0.3 \mu \mathrm{m}$, and 21.9 
\pm SE $0.7 \mu \mathrm{m}$, respectively. For $n=1.3611$, the $L_{\mathrm{c}}$ of the BFRL are calculated to be $66.9 \pm$ SE $1.2 \mu \mathrm{m}$ (with MCF-10A), $27.9 \pm$ SE $0.7 \mu \mathrm{m}$ (with MCF-7), and 50.5 \pm SE $1.6 \mu \mathrm{m}$ (with MDA-MB-231), respectively. The BFRL filled with MCF-7 cells has the smallest laser cavity, whereas the BFRL filled with MCF-10A cells has the largest laser cavity. The size of the laser cavity is associated with scattering strength in the random laser system. The laser cavity with small size means that the scattering strength in random laser system is strong, and vice versa. Based on the previous discussion regarding to the lasing threshold, the BFRL with MCF-10A shows weakest scattering strength among the three BFRLs, which is consistent with the PFT results. However, it should be noticed that the PFT results show obvious different between the BFRLs with $\operatorname{MCF}-7\left(p_{m=1}=12.1 \mu \mathrm{m}\right)$ and MDA-MB-231 cells $\left(p_{m=1}=21.9 \mu \mathrm{m}\right)$. This is because the PFT analysis extracts and reveals the small variation in the lasing spectra (e.g. wavelength shift). The calculated cavity sizes are bigger than the individual cells. It means the lasing cavity is formed by several cells, which comprises both the light path in the gain medium and inside the cells. As mentioned in pervious discussion, the light path inside the MDA-MB-231 cells are longer than other cases due to their subcellular biophysical structure. The light travels inside the cells cannot experience gain amplified. Thus, the light path in the gain medium should be longer to obtain sufficient gain to lase, which results in a large laser cavity in the BFRL with MDA-MB-231 cells. This means that the PFT results are in good agreement with above findings based on the lasing spectra. This suggests that our BFRL can be used to identify the different biophysical properties and consequently distinguish normal and cancerous cells in liquid suspensions via analyzing the random lasing properties.

Beside biophysical phenotyping of the cell suspensions, extending the BFRL to the detection of cancerous cells in cell mixtures is achievable by comprehensively investigating the BFRL properties of biosamples. The experiment details can be found in the Supporting Information: Cell type classification and detection of floating cancerous cells using BFRL. A linear combination of the three BRFL properties (i.e., threshold, peak shift, and optical path length of fundamental Fourier component $p_{m=1}$ ), $0.68 \times$ threshold $+0.10 \times$ peak shift $+0.73 \times$ optical path length, was found to be a good 
indicator to classify the types of cells and detect the cancerous cells in cell mixtures, as shown in Figure S5. Thus, the BFRL could be further developed for cell type detection based on the biophysical phenotyping.

\section{Conclusion}

In summary, we have presented a label-free random laser cytometer with the sensing strategies of the different lasing properties tuned by the whole-cell as well as the subcellular biophysical properties of the single floating cells. Three key parameters were proposed in the BFRL, i.e. lasing threshold, wavelength shift and optical pathlength. The lasing threshold was demonstrated strongly relevant to the whole-cell scattering of the biofluids. On the other hand, the peak shift of the lasing spectra was strongly influenced by the subcellular structure of the cells, i.e. cortical shells and nuclear lamina shells. The PFT analysis further confirm the influence of the cellular and subcellular structure on the random lasing properties in the BFRL, leading to a sensitive and parameterized sensing strategy. By performing more measurements with different batches of microfluidic devices and cells, the repeatability and reliability of our BFRL is verified. A cell type classification strategy is introduced by adapting principle component analysis, and the first order principle component is shown a good indicator for detecting cancerous cells in cell mixtures. Our work extends the biological random laser system to integrate with optofluidic devices and reveals the possibility of the random-laser-based cytometer, which opens a new way for cancerous cell diagnostic using random laser.

\section{Acknowledgement}

This work was supported by the Central Research Fund Project No. PolyU 152207/15E, PolyU Central Grant project 1-ZVGB, General Program of National Natural Science Foundation of China (project\# 31500758), Croucher Foundation Startup Grant, City

University of Hong Kong Research Grant (project\# 7004602), General Research Grant 
(project\# 11206014), Collaborative Research Fund (project\# C1013-15GF) of Hong Kong Research Grant Council, H2020 Marie Skłodowska-Curie Actions (MSCA) (744817) and China Postdoctoral Science Foundation (2015M571918, 2017T100442).

Supporting Information Available: The following files are available free of charge.

BFRL_SI.docx. The sensitivity analysis, the influences of cell density on the random laser properties, repeatability and reliability of the BFRL, and cell type classification and detection of floating cancerous cells using BFRL.

\section{Reference}

(1) Psaltis, D.; Quake, S. R.; Yang, C. Developing Optofluidic Technology through the Fusion of Microfluidics and Optics. Nature 2006, 442 (7101), 381-386.

(2) Fan, X.; White, I. M. Optofluidic Microsystems for Chemical and Biological Analysis. Nat. Photonics 2011, 5 (10), 591-597.

(3) Erickson, D.; Mandal, S.; Yang, A. H. J.; Cordovez, B. Nanobiosensors: Optofluidic, Electrical and Mechanical Approaches to Biomolecular Detection at the Nanoscale. Microfluid. Nanofluidics 2008, 4 (1-2), 33-52.

(4) Minzioni, P.; Osellame, R.; Sada, C.; Zhao, S.; Omenetto, F. G.; Gylfason, K. B.; Haraldsson, T.; Zhang, Y.; Ozcan, A.; Wax, A.; et al. Roadmap for Optofluidics. J. Opt. 2017, 19 (9), 093003-093052.

(5) Reynolds, T.; Riesen, N.; Meldrum, A.; Fan, X.; Hall, J. M. M.; Monro, T. M.; François, A. Fluorescent and Lasing Whispering Gallery Mode Microresonators for Sensing Applications. Laser Photon. Rev. 2017, 11 (2), 1600265-1600284.

(6) Huang, N.-T.; Zhang, H.; Chung, M.-T.; Seo, J. H.; Kurabayashi, K. Recent Advancements in Optofluidics-Based Single-Cell Analysis: Optical on-Chip Cellular Manipulation, Treatment, and Property Detection. Lab Chip 2014, 14 (7), 1230-1245.

(7) Fan, X.; Yun, S.-H. The Potential of Optofluidic Biolasers. Nat. Methods 2014, 11 (2), 141147.

(8) Chen, Y.-C.; Chen, Q.; Fan, X. Lasing in Blood. Optica 2016, 3 (8), 809-815.

(9) Chen, Y.-C.; Chen, Q.; Fan, X. Optofluidic Chlorophyll Lasers. Lab Chip 2016, 16 (12), 22282235.

(10) Sun, Y.; Shopova, S. I.; Wu, C.-S.; Arnold, S.; Fan, X. Bioinspired Optofluidic FRET Lasers via DNA Scaffolds. Proc. Natl. Acad. Sci. 2010, 107 (37), 16039-16042.

(11) Gather, M. C.; Yun, S. H. Single-Cell Biological Lasers. Nat. Photonics 2011, 5 (7), 406-410.

(12) Yang, Y.; Liu, A. Q.; Lei, L.; Chin, L. K.; Ohl, C. D.; Wang, Q. J.; Yoon, H. S. A Tunable 3D Optofluidic Waveguide Dye Laser via Two Centrifugal Dean Flow Streams. Lab Chip 2011, 11 
(18), 3182-3187.

(13) Balslev, S.; Kristensen, A. Microfluidic Single-Mode Laser Using High-Order Bragg Grating and Antiguiding Segments. Opt. Express 2005, 13 (1), 344-351.

(14) Vannahme, C.; Smith, C. L. C.; Brøkner Christiansen, M.; Kristensen, A. Emission Wavelength of Multilayer Distributed Feedback Dye Lasers. Appl. Phys. Lett. 2012, 101 (15), 151123-151126.

(15) Shivakiran Bhaktha, B. N.; Bachelard, N.; Noblin, X.; Sebbah, P. Optofluidic Random Laser. Appl. Phys. Lett. 2012, 101 (15), 1-5.

(16) Wiersma, D. S. The Physics and Applications of Random Lasers. Nat. Phys. 2008, 4 (5), 359367.

(17) Cao, H. Lasing in Random Media. Waves in Random Media 2003, 13 (3), R1-R39.

(18) Luan, F.; Gu, B.; Gomes, A. S. L.; Yong, K. T.; Wen, S.; Prasad, P. N. Lasing in Nanocomposite Random Media. Nano Today 2015, 10 (2), 168-192.

(19) Viola, I.; Ghofraniha, N.; Zacheo, A.; Arima, V.; Conti, C.; Gigli, G. Random Laser Emission from a Paper-Based Device. J. Mater. Chem. C 2013, 1 (48), 8128-8133.

(20) Bachelard, N.; Gigan, S.; Noblin, X.; Sebbah, P. Adaptive Pumping for Spectral Control of Random Lasers. Nat. Phys. 2014, 10 (6), 426-431.

(21) Polson, R. C.; Vardeny, Z. V. Random Lasing in Human Tissues. Appl. Phys. Lett. 2004, 85 (7), 1289-1291.

(22) Polson, R. C.; Vardeny, Z. V. Organic Random Lasers in the Weak-Scattering Regime. Phys. Rev. B - Condens. Matter Mater. Phys. 2005, 71 (4), 37-41.

(23) Song, Q.; Xiao, S.; Xu, Z.; Liu, J.; Sun, X.; Drachev, V.; Shalaev, V. M.; Akkus, O.; Kim, Y. L. Random Lasing in Bone Tissue. Opt. Lett. 2010, 35 (9), 1425-1427.

(24) Wang, C.-S.; Chang, T.-Y.; Lin, T.-Y.; Chen, Y.-F. Biologically Inspired Flexible QuasiSingle-Mode Random Laser: An Integration of Pieris Canidia Butterfly Wing and Semiconductors. Sci. Rep. 2014, 4 (1), 6736-6741.

(25) Dominguez, C. T.; Lacroute, Y.; Chaumont, D.; Sacilotti, M.; de Araújo, C. B.; Gomes, A. S. L. Microchip Random Laser Based on a Disordered TiO2-Nanomembranes Arrangement. Opt. Express 2012, 20 (16), 17380-17385.

(26) Wang, Y.; Duan, Z.; Qiu, Z.; Zhang, P.; Wu, J.; Zhang, D.; Xiang, T. Random Lasing in Human Tissues Embedded with Organic Dyes for Cancer Diagnosis. Sci. Rep. 2017, 7 (1), 8385-8391.

(27) Steinkamp, J. A.; Stewart, C. C.; Crissman, H. A. Three-Color Fluorescence Measurements on Single Cells Excited at Three Laser Wavelengths. Cytometry 2005, 2 (4), 226-231.

(28) He, J.; Chan, W.-K.; Cheng, X.; Tse, M.-L.; Lu, C.; Wai, P.-K.; Savovic, S.; Tam, H.-Y. Experimental and Theoretical Investigation of the Polymer Optical Fiber Random Laser with Resonant Feedback. Adv. Opt. Mater. 2018, 1701187-1701195.

(29) Hu, Z.; Zhang, Q.; Miao, B.; Fu, Q.; Zou, G.; Chen, Y.; Luo, Y.; Zhang, D.; Wang, P.; Ming, H.; et al. Coherent Random Fiber Laser Based on Nanoparticles Scattering in the Extremely Weakly Scattering Regime. Phys. Rev. Lett. 2012, 109 (25), 2-6.

(30) Wang, Y.; Ta, V. D.; Gao, Y.; He, T. C.; Chen, R.; Mutlugun, E.; Demir, H. V.; Sun, H. D. Stimulated Emission and Lasing from CdSe/CdS/ZnS Core-Multi-Shell Quantum Dots by Simultaneous Three-Photon Absorption. Adv. Mater. 2014, 26 (18), 2954-2961.

(31) Li, X.; Wang, Y.; Sun, H.; Zeng, H. Amino-Mediated Anchoring Perovskite Quantum Dots for 
Stable and Low-Threshold Random Lasing. Adv. Mater. 2017, 29 (36), 1701185-1701193. Liang, X. J.; Liu, A. Q.; Lim, C. S.; Ayi, T. C.; Yap, P. H. Determining Refractive Index of Single Living Cell Using an Integrated Microchip. Sensors Actuators A Phys. 2007, 133 (2), 349-354.

(33) Meng, X.; Fujita, K.; Murai, S.; Konishi, J.; Mano, M.; Tanaka, K. Random Lasing in Ballistic and Diffusive Regimes for Macroporous Silica-Based Systems with Tunable Scattering Strength. Opt. Express 2010, 18 (12), 12153-12160.

(34) El-Dardiry, R. G. S.; Mooiweer, R.; Lagendijk, A. Experimental Phase Diagram for Random Laser Spectra. New J. Phys. 2012, 14 (11), 113031-113041.

(35) Wu, X. H.; Yamilov, A.; Noh, H.; Cao, H.; Seelig, E. W.; Chang, R. P. H. Random Lasing in Closely Packed Resonant Scatterers. J. Opt. Soc. Am. B 2004, 21 (1), 159-167.

(36) Hu, Z.; Xia, J.; Liang, Y.; Wen, J.; Miao, E.; Chen, J.; Wu, S.; Qian, X.; Jiang, H.; Xie, K. Tunable Random Polymer Fiber Laser. Opt. Express 2017, 25 (15), 18421-18430.

(37) Watanabe, E.; Hoshiba, T.; Javidi, B. High-Precision Microscopic Phase Imaging without Phase Unwrapping for Cancer Cell Identification. Opt. Lett. 2013, 38 (8), 1319-1321.

(38) Hu, Z.; Gao, P.; Xie, K.; Liang, Y.; Jiang, H. Wavelength Control of Random Polymer Fiber Laser Based on Adaptive Disorder. Opt. Lett. 2014, 39 (24), 6911-6914.

(39) Veltri, A.; Infusino, M.; Ferjani, S.; De Luca, A.; Strangi, G. Blue-Shifted Random-LaserMode Selection in Gain-Assisted Anisotropic Complex Fluids. Phys. Rev. E - Stat. Nonlinear, Soft Matter Phys. 2011, 83 (4), 1-6.

(40) Wu, Y.; Ren, Y.; Chen, A.; Chen, Z.; Liang, Y.; Li, J. J.; Lou, G.; Zhu, H.; Gui, X.; Wang, S.; et al. One-Dimension Random Laser Based on Artificial High-Index Contrast Scatterers. Nanoscale 2017, 9 (21), 6959-6964.

(41) Mani, S. A.; Guo, W.; Liao, M.-J.; Eaton, E. N.; Ayyanan, A.; Zhou, A. Y.; Brooks, M.; Reinhard, F.; Zhang, C. C.; Shipitsin, M.; et al. The Epithelial-Mesenchymal Transition Generates Cells with Properties of Stem Cells. Cell 2008, 133 (4), 704-715.

(42) Consoli, A.; López, C. Lasing Optical Cavities Based on Macroscopic Scattering Elements. Sci. Rep. 2017, 7 (1), 40141-40147.

(43) Consoli, A.; Mariano da Silva, D.; Wetter, N. U.; López, C. Large Area Resonant Feedback Random Lasers Based on Dye-Doped Biopolymer Films. Opt. Express 2015, 23 (23), 2995429963.

(44) Sharma, D.; Ramachandran, H.; Kumar, N. Lévy Statistics of Emission from a Novel Random Amplifying Medium: An Optical Realization of the Arrhenius Cascade. Opt. Lett. 2006, 31 (12), 1806-1808.

(45) Lin, X.; Wan, N.; Weng, L.; Zhou, Y. Light Scattering from Normal and Cervical Cancer Cells. Appl. Opt. 2017, 56 (12), 3608-3614.

(46) Bereiter-Hahn, J.; Fox, C. H.; Thorell, B. Quantitative Reflection Contrast Microscopy of Living Cells. J. Cell Biol. 1979, 82 (3), 767-779. 


\section{Figures}

(a)

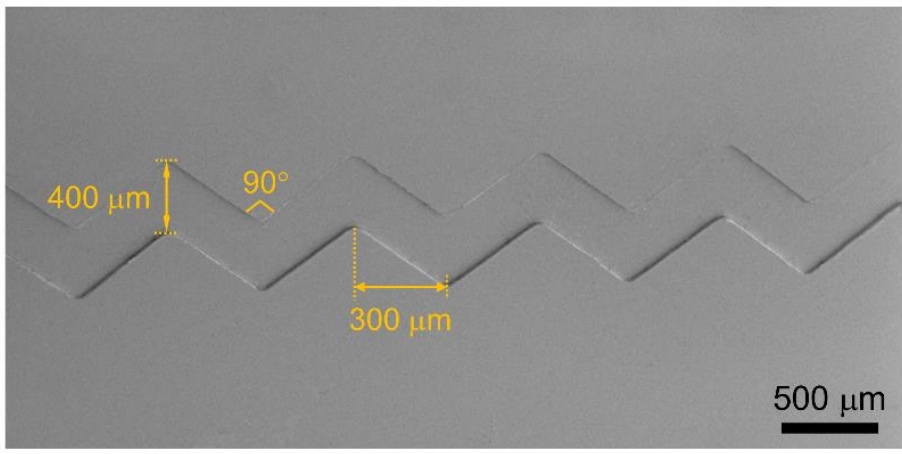

(b)

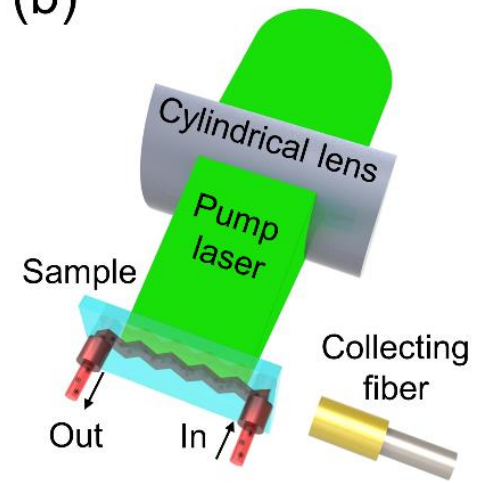

Figure 1. (a) SEM image of the zigzag channel in the BFRL. The detailed structure parameters are indicated in the image. (b) Schematic of the pump and collection configuration.

(a)

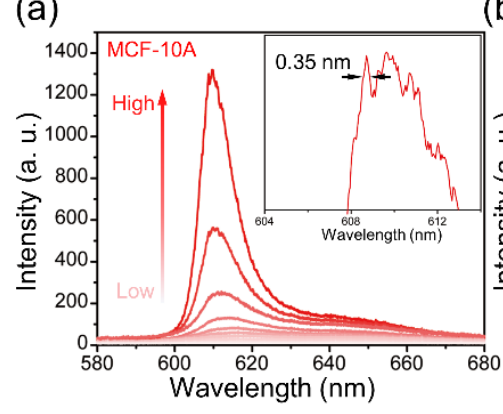

(b)

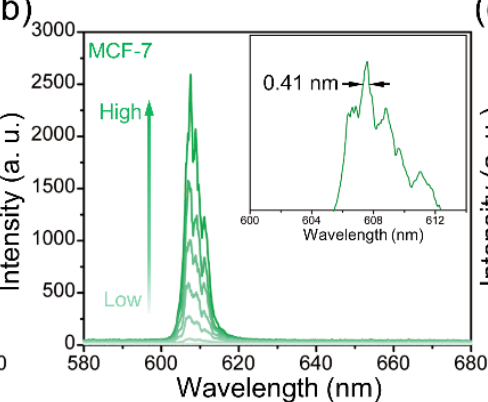

(c)

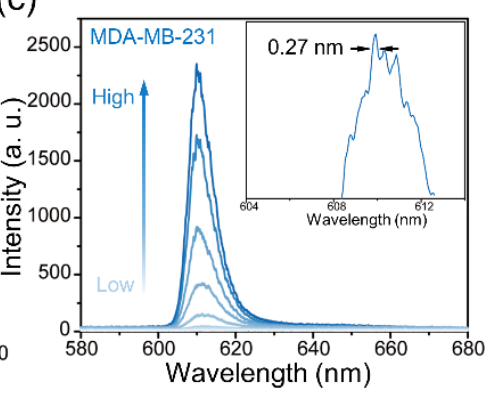

Figure 2. Lasing spectra of the BFRL filled with (a) MCF-10A, (b) MCF-7, and (c) MDA-MB-231 cells with same cells concentration at different pump powers. The insert in each figure shows the zoom-in lasing peaks. The FWHM of the main lasing peaks is marked. 


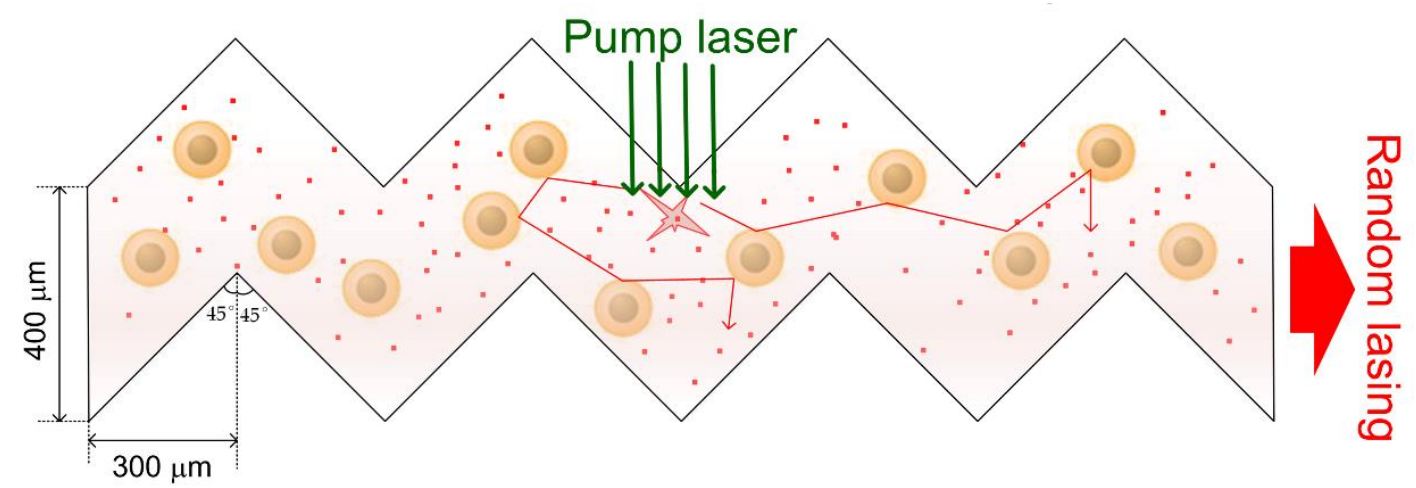

Figure 3. Schematic of the lasing mechanism of the BFRL. The suspended cells introduced large amounts of scattering events, which initiates the random laser.

(a) MCF-10A

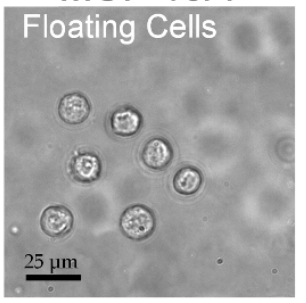

(c)

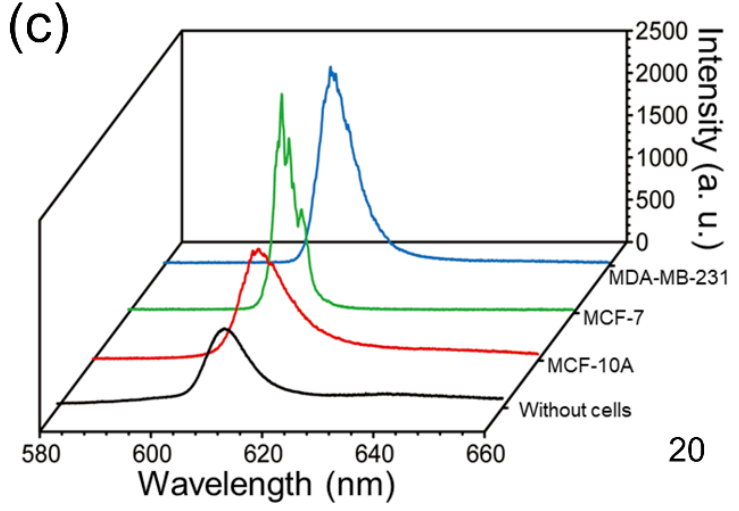

MCF-7

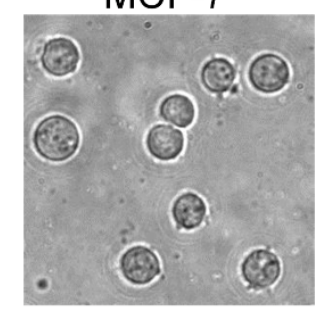

20
MDA-MB-231 (b)

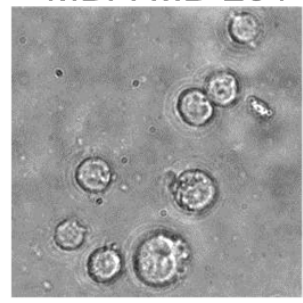

(d)

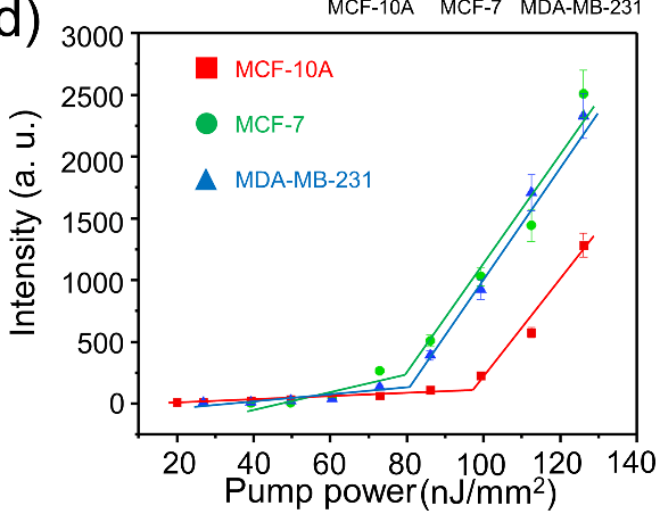

Figure 4. (a) Optical images of MCF-10A, MCF-7, and MDA-MB-231 cells. (b) Diameters of MCF-10A, MCF-7, and MDA-MB-231 cells. (c) Emission spectra of the BFRL with three different cells and without cells at the same pump power. (d) The lasing intensity as a function of pump power of the BFRL filled with three different cells. * indicates statistical significance at a level of $5 \%$. 

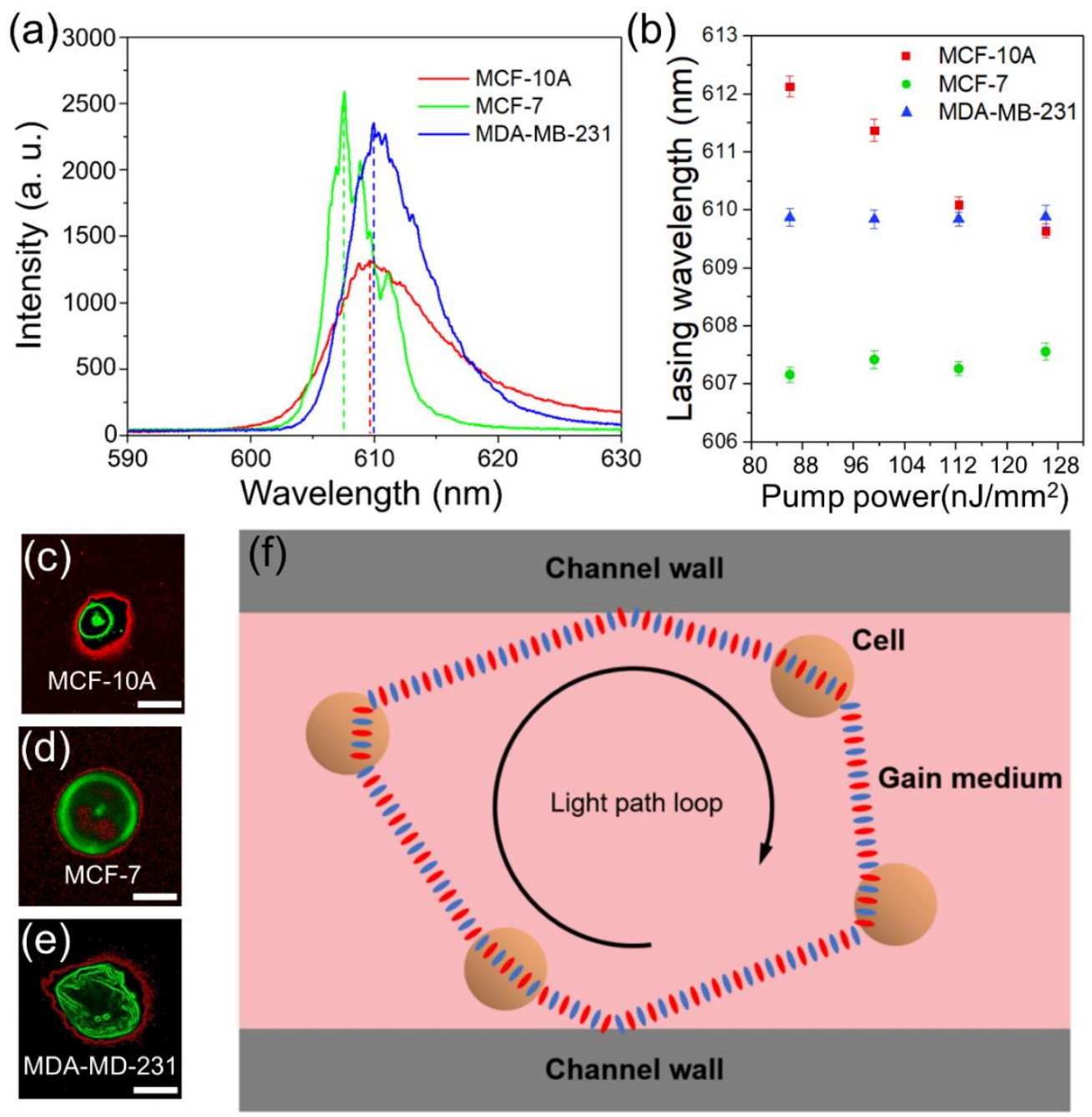

Figure 5. (a) Lasing spectra of BFRL filled with MCF-10A cells (red curve), MCF-7 cells (green curve), and MDA-MB-231 cells (blue curve) at the pump power of 126 $\mathrm{nJ} / \mathrm{mm}^{2}$. (b) The lasing wavelength of BFRL filled with MCF-10A cells (red square), MCF-7 cells (green circle), and MDA-MB-231 cells (blue triangle) as a function of pump power. (c-e) Immunostaining of the three types of cells. The cortical actin (red) and the nuclear lamina Lamin-A (green) represent the shell structures of the cell. The scale bar indicates $10 \mu \mathrm{m}$. (f) Schematic of the light path loop in BFRL. The pink background represents the solution contains gain medium. The brown circles represent the cells. The light path is indicated by the wavefronts as the intercalated red and blue ellipses. 


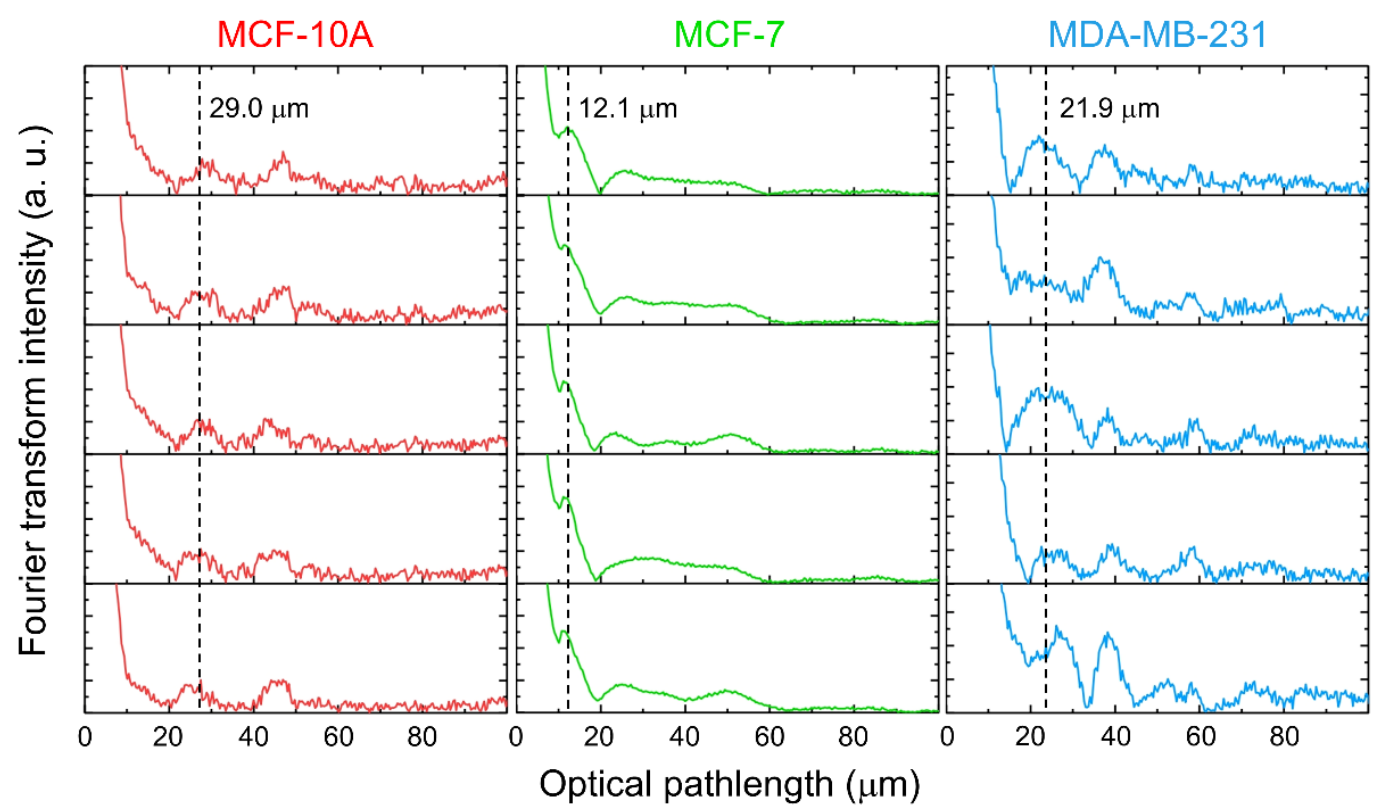

Figure 6. The power Fourier transform results of the lasing spectra of the BFRL with three different cells. The optical pathlengths of the first order Fourier harmonic are listed in each curve. For each sample, five spectra from five identical measurements are analyzed. 


\section{for TOC only}

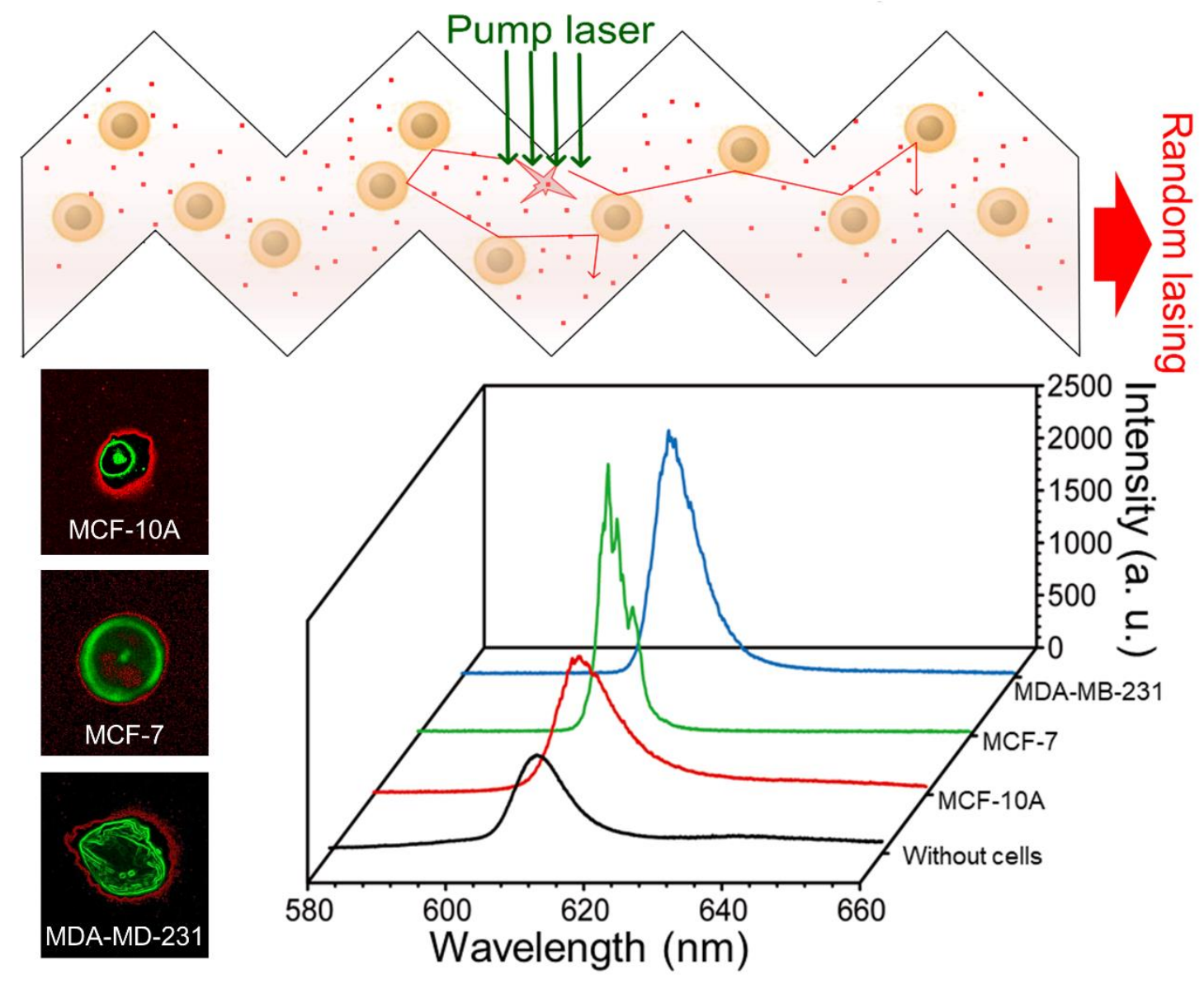

